\title{
Analysis of Optofluidic Ring-Resonator for Bio-sensing Application
}

\author{
SuhasKamuni, Anishka Divakaran, Adarsh M M, Swekrithi Shetty and Shwetha MSai \\ Vidya Institute of Technology, Bengaluru, India - 560064
}

\begin{abstract}
In this paper, an optofluidic ring resonator with the all-pass configuration is designed. The structure has a bus waveguide and two ring waveguides, in which one ring is used as a reference ring, while the other is used as sensing ring.The designed ring resonator structure will detect a resonance shift, when a $10 \%$ glucose solution as used as analyte. Which would be useful in bio-sensing application.
\end{abstract}

Keywords: Optofluidic Ring, Bio Sensing, Bus Waveguide

\section{INTRODUCTION}

Lab-on-a-chip (LOC) is one important technology which helps in the label-free detection of a particular disease in a short period of time. The use of silicon in photonics is one among the many technologies, the fastest growing industries for LOC applications. Ring resonators based on Silicon-OnInsulator (SOI) technology is a major contributor for the purpose of biosensing. This is because silicon allows structures of even the smallest size to be used, when designed with the correct dimensions. On the principle of resonance, the ring resonator works; it depends on the resonator length. The devices used in medical research have undergone a radical transformationin detecting various diseases with various methodologies.

Over the last few years, advances in various real-time sensing applications have come by virtue of improvements in computational speeds or breakthroughs achieved by miniaturization of devices. Since sensors are employed in almost every device used today, it is essential to make them efficient without compromising on the quality or increasing the costs [1]. Since manufacturing of integrated sensors in different configurations can be done, they can be implemented in various industries like the automobile industry, defense industry, healthcare industry, etc. [2]. Ring resonators came to the fore in 1969 , when it was presented to the world by $\mathrm{P}$ Troughton. In the field of optics, it is feasible to work on micro-ring resonators due to the ease of fabrication and high sensitivity. Another important characteristic is quality factor, which makesit a top favorite for biosensing related work [3].

Labeled and label free detections are the two methods, which can be implemented in the design for the detection of biomolecules. Labeled detection has an intermediate step which complicates the detection process of the sample prepared. The label can alter the binding properties of the molecule thereby reducing the reliability of detection. Labelfree sensors strive to overcome the stability and reliability problems faced in labeled devices. Label-free detection schemes are able to directly detect the required molecules as they bind in real time [4]. Label-free sensing technologies are a field where developments are keenly studied for both research and industrial interests.

In integrated optical sensors, detection is based on the measurement of change in the refractive index of the material and wavelength on which the device operates. Light of specific wavelengths is confined, and the refractive index changes when molecules bind to the surface of the device. The effectiveness of a bio-sensor is determined by its resolution and the resolution depends on the detection limit. Detection limit is defined as the lowest amount of analyte that can be detected from the given sample and the dimensions of the ring structure is a key factor for determining the detection limit. Silicon is still a favored material for manufacturing integrated devices asthey can be fabricatedat extremely low costs, and are disposable in nature, thereby eliminating need for cleaning them [5].

\section{DESIGN}

A two Port dual micro-ring resonator model is been designed. An all pass configuration of double microring resonator is preferred due to its high quality factor when compared with the add drop configuration. The two Port dual micro-ring resonator has two identical rings in parallel configuration, which are independently coupled to the bus waveguide and also to each other. The ring structure includes an inner radius of $2.7 \mu$ mand an outer radius of $3.1 \mu \mathrm{m}$. There exists two ring-bus coupling and one ring-ring coupling region.

The distance between the rings and the bus waveguide is $0.1 \mu \mathrm{m}$ and the distance between the two rings is $0.1 \mu \mathrm{m}$. The length of the bus waveguide is $25 \mu \mathrm{m}$, with the thickness of $0.18 \mu \mathrm{m}$ and width of $0.4 \mu \mathrm{m}$. The material used for the bus waveguide and the ring are silicon. The substrate used is made up of $\mathrm{SiO}_{2}$, with a thickness of is $4 \mu \mathrm{m}$. An opto-fluidic ring-resonator structure simulated using Lumerical FDTD solution software is depicted in figure 1 .

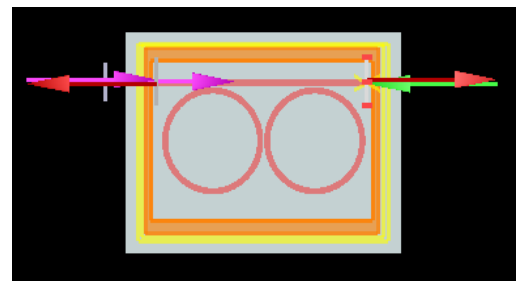

Figure 1: Ring resonator structure without the analyte. 
Figure 2 depicts the ring resonator structure with $10 \%$ glucose concentration in the first ring, which is highlighted in the figure 2 , and is used a analyte for biosensing application.

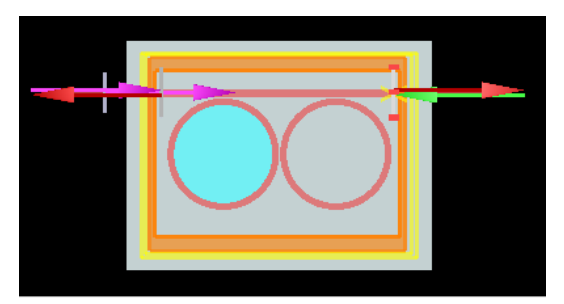

Figure 2: Dual ringstructure with $10 \%$ glucose concentration in the first ring.

\section{THEORY}

The dual ring configuration of ring resonator consists of two rings arranged in either series or in parallel. In a series configuration, the waveguides which are looped backie. The ring waveguides are also coupled to one another and the signal is passed from the input port to the output port sequentially through both the rings whereas in a parallel configuration shown in figure 1 , has the rings individually coupled to both input and throughput ports which may or may not be individually coupled to each other. The input signal is passed simultaneously through both the rings, which results in non-identical resonance peaks, thus allowing a multiple detection. Therefore, for bio-sensing applications, one of the rings is used as the sensing ring, and the other is used as the reference ring.

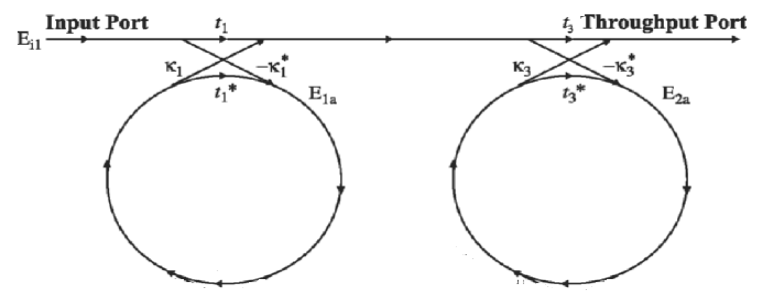

Figure 3: Parallel Configuration of Dual Ring Resonator

Figure 3 depicts the parallel configuration of ring resonator structure with all pass configuration. A source with wavelength in mid infrared region ranging from $1500 \mathrm{~nm}$ to $1600 \mathrm{~nm}$ is given to the input bus waveguide. The light interaction in the ring resonator can be described in matrix form and is given as follows: [6].

$$
\left(\begin{array}{l}
E_{t 1} \\
E_{t 2}
\end{array}\right)=\left(\begin{array}{cc}
t & \kappa \\
-\kappa^{*} & t^{*}
\end{array}\right)\left(\begin{array}{l}
E_{i 1} \\
E_{i 2}
\end{array}\right)
$$

Where $\mathrm{E}$ is the complex mode amplitude, $\mathrm{t}$ and $\mathrm{k}$ are the coupler parameters, $\mathrm{t}^{*}$ and $\mathrm{k}^{*}$ are the conjugate complex values of $t$ and $k$.

The analyte placed in one of thecavityof ring waveguide which is placed on the substrate causes a change in the refractive index, which in turn changes the transmission behaviour of the propagating light in the micro-ring. This change in a micro-ring resonator can be monitored by the resonance condition given by the equation,

$$
2 \pi \mathrm{RN}_{\mathrm{eff}}=\mathrm{m} \lambda_{\mathrm{r}} \ldots \ldots \ldots
$$

Where $\mathrm{N}_{\text {eff }}$ is the refractive index, $\lambda{ }_{\mathrm{r}}$ is the resonance wavelength, $\mathrm{m}$ is an integer, $\mathrm{R}$ is the radius of the ring structure.

Q-factor is one of the important parameters which influences the bio-sensing ability of the ring resonator. It is defined as the ratio of the operational wavelength to the resonance width. Mathematically, it can be written as:

$$
\mathrm{Q}=\lambda / 2 \delta \lambda \ldots \ldots \ldots \ldots
$$

Where, $2 \delta \lambda$ is full width half maximum (FWHM) defined as the $3 \mathrm{~dB}$ bandwidth of the resonance. FWHM is given by:

$$
2 \delta \lambda=\lambda^{2}\left(1-\mathrm{t}^{2}\right) / \pi \mathrm{N} \text { eff } \mathrm{t}
$$

From (4),

$$
\mathrm{Q}=\pi \operatorname{Neff} \mathrm{L} \mathrm{t} / \lambda\left(1-\mathrm{t}^{2}\right)
$$

Number of round trips made by energy before being lost to bus waveguide is also important to determine the $\mathrm{Q}$ factor, hence for better sensing the round-trip losses and coupling in directional coupler should be low, this in turn will improve the $\mathrm{Q}$ factor [7].

\section{RESULt}

An All pass configuration of double ring resonator is designed for bio-sensing applications. The rings are arranged in parallel to each other and are independently coupled to the bus waveguide. A $10 \%$ concentration of glucose-water solution is used as analyte to analyse the changes in the spectral properties by comparing the design with and without the analyte on one of the ring waveguides.

In the figure 4 light confinement from the bus waveguide to the ring waveguide can be observed for a wavelength of $1.6 \mu \mathrm{m}[8]$.

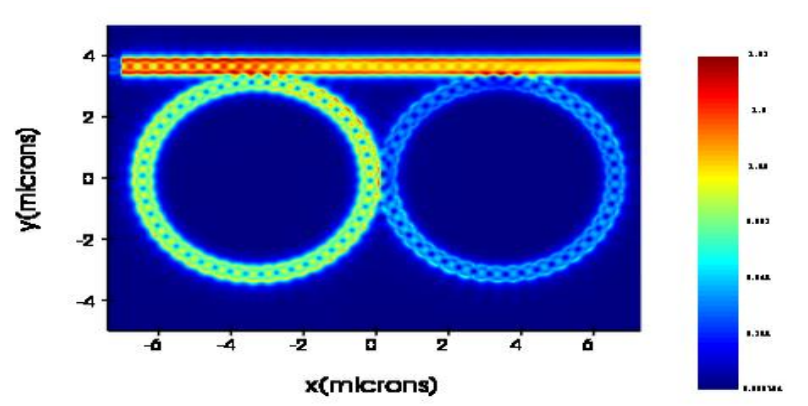

Figure 4: Light confinement in the rings

When the liquid analyte is placed on the ring waveguide, a shift in wavelength can be observed. This shift in 
wavelength is caused when there is a change in cladding due to the introduction of analyte, this further changes the effective refractive index of the core and cladding [9]. The sensitivity of the device can be thus defined by observing the shift in the wavelength.

The transmittance plot versus wavelength in $\mu \mathrm{m}$ ranging from $1.5 \mu \mathrm{m}$ to $1.6 \mu \mathrm{m}$ is observed in the figure 5 . The figure 5(a) gives a comparison of transmittance of light between the input port 1 and output through port2. The inequality of the resonance peak is observed to analyze the changes in the intensity of light confined in the ring through the bus waveguide.

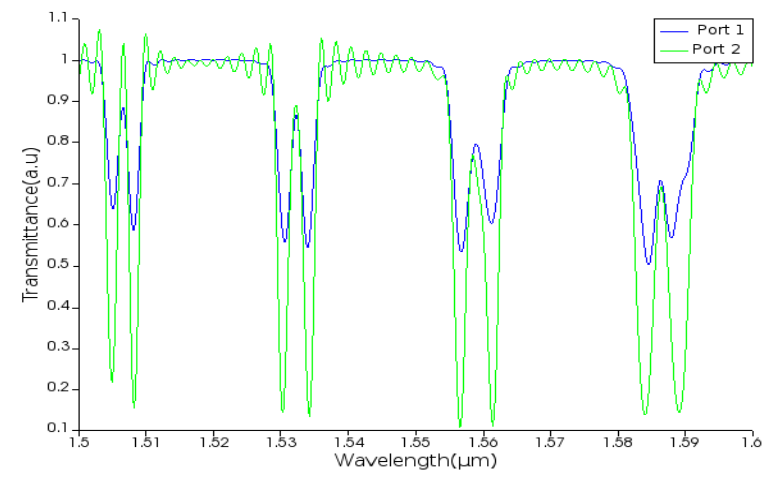

Figure 5(a):Transmittance plot without the analyte

Similarly, the figure 5(b) gives a comparison of transmittance of light between port1 and port 2 when a liquid analyte i.e., $10 \%$ glucose-water concentration is placed on the first ring.The figure 5(a)\& 5(b) are compared to analyze the changes in the behavior of transmittance of light within the waveguide for sensing applications.

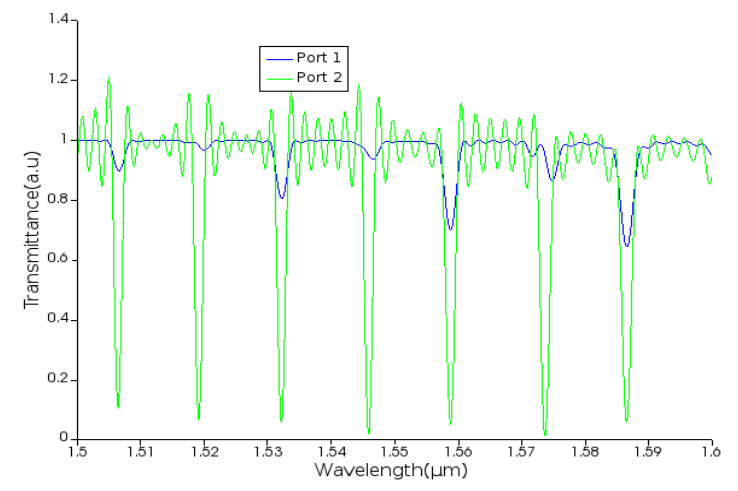

Figure 5(b): Transmittance plot with the liquid analyte

The power at input port1 and the output port 2 can be observed from the power plot in the figure 6(a). It can be analyzed that there is an increase in the power from input to output port which is considered to be one of the crucial parameters in sensing. The power plot is further analyzed for port 1 and port 2 after placing an analyte onto the first ring as shown in figure 6(b).

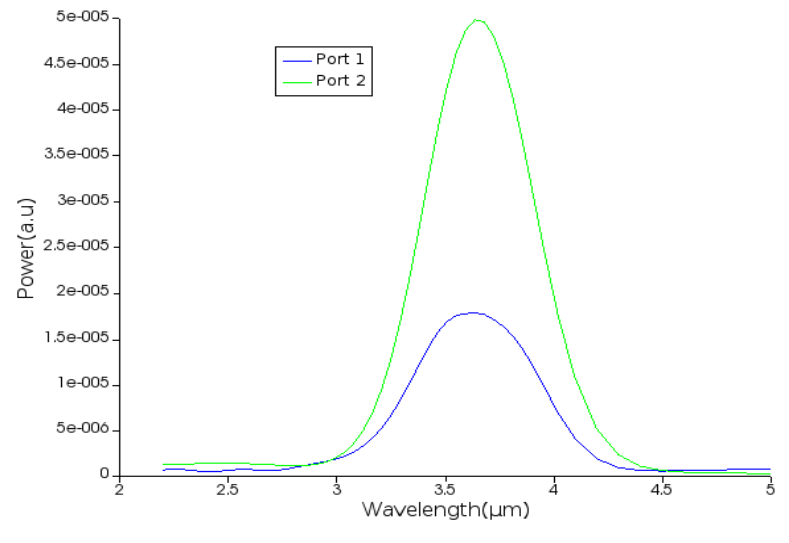

Figure 6(a): Power plot without glucose concentration.

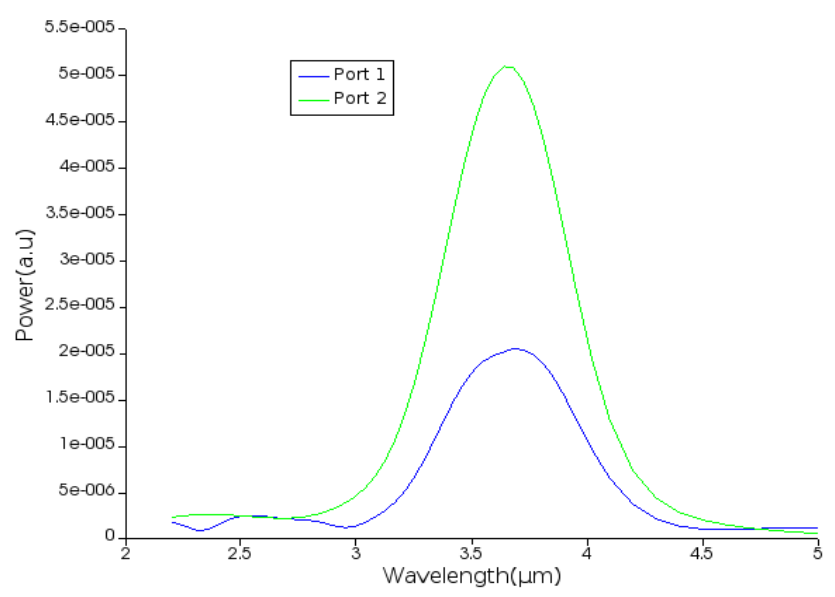

Figure 6(b) : Power plot with $10 \%$ glucose concentration.

\section{CONCLUSION}

In this paper, a double ring opto-fluidic ring resonator is designed for bio-sensing applications. The structure consists of two waveguides looped back to form a ring structure and a rectangular bus waveguide, known as all-pass configuration and is preferred over add drop configuration due to its better Q-factor.Coupling of light is observed between the bus waveguide and the ring structures for a range of wavelength varying from $1.5 \mu \mathrm{m}$ to $1.6 \mu \mathrm{m}$. For biosensing applications a resonance shift is observed when $10 \%$ glucose concentration is placed in one of the cavity of the ring resonator structure.

\section{ACKNOWLEDGMENT}

This work is supported by Vision Group on Science and Technology, Department of IT, BT \& ST, Government of Karnataka, India. Grant number: VGST - CISEE GRD 466. 


\section{REFERENCES}

[1] H.K Hunt, Andrea M. Armani, Label- free Biological and Chemical Sensors, Nanoscale 2010, 2,1544-1559

[2] J. Carrano, Chemical and Biological Sensor Standards Study, DARPA: Arlington, VA, USA, 2005, p. 36

[3] Zhixian Xia, Zhiping Zhou, Sensitivity Analysis of Microring Resonator Based Biosensor: The Quality Factor Perspective, 2006 IEEE, 1-4244-0816-4/06

[4] Simin Mehrabani, Ashley J. Maker, Andrea M. Armani, Hybrid Integrated Label- Free Chemical and Biological Sensors, Sensors 2014, 14, 5890-5928

[5] Tom Claes, Jordi Gironès Molera, Katrien De Vos, Etienne Schacht, Roel Baets, Peter Bientsman, Label-free Biosensing With a Slot- Waveguide-Based Ring Resonator in Silicon on Insulator, IEEE Photonics Journal, Vol. 1, No. 3, September 2009

[6] Dominik G Rabus., [Integrated Ring Resonators, The Compendium], Springer Series in Optical Sciences, SpringerVerlag Berlin Heidelberg, 3-34(2007).

[7] Wim Bogaerts*, Peter De Heyn, Thomas Van Vaerenbergh, Katrien DeVos, Shankar Kumar

[8] Selvaraja, Tom Claes, Pieter Dumon, Peter Bienstman, Dries Van Thourhout, and Roel Baets,Silicon microring resonators, Laser Photonics Rev. 6, No. 1, 47-73 (2012).

[9] Shwetha M, Navya Krishna Reddy, Prasant Kumar Pattnaik, Narayan K," Design and analysis of silicon ring resonator for bio-sensing application". Proc. of SPIEVol.10690, (2018).

[10] Patrick Steglich, Marcel Hülsemann, Birgit Dietzel and Andreas Mai," Optical Biosensors Based on Silicon-On-Insulator Ring Resonators", Molecules 2019, 24, 519. 